\title{
OPTICAL REDSHIFT SURVEYS
}

\author{
L. Nicolaci da Costa \\ Harvard-Smithsonian Center for Astrophysics and CNPq/Observatório Nacional \\ 60 Garden Street, Cambridge, MA 02138, U.S.A.
}

\section{INTRODUCTION}

Redshift surveys of galaxies have been over the past decade the major source of information for studies of the large-scale structure of the Universe. Following the completion of the original CfA Redshift Survey, several groups have joined the endeavor, probing different regions of the sky to different depths in a remarkable long-term effort to study the nature of the galaxy distribution and its statistical properties at different scales. Here I summarize the current status of the ongoing surveys drawn from optical galaxy catalogs. The review is not intended to be complete but rather to demonstrate the vitality of the area and to point out that exciting new data should be forthcoming in the next few years.

\section{NEARBY SURVEYS}

For galaxies brighter than magnitude 14.5, surveys such as the CfA1 (Huchra et al. 1983), Arecibo (Haynes and Giovanelli 1986), nearby galaxies (Tully and Fisher 1987), SSRS (da Costa et al. 1991) and its recent extensions to cover the equatorial region in the interval $-17.5^{\circ}<\delta<0^{\circ}$ (Fairall et al. 1991, Huchra et al. 1991) and SPS (Dressler 1991) have provided essentially complete redshift data for both galactic caps, and well sampled regions at low galactic latitudes. Recently, an attempt has been made to combine these data into a homogeneous ' all-sky' magnitude-limited sample (Willmer et al. 1991). Despite the uncertainties regarding the degree of homogeneity, this 'all-sky' sample provides for the first time an unbiased view of the galaxy distribution out to about $8,000 \mathrm{~km} \mathrm{~s}^{-1}$. In the southern hemisphere, for instance, it shows remarkably well the existence of wall-like features in our neighborhood like the Southern wall, the Telescopium-Pavo-Indus (TPI) complex, and the supergalactic plane, which extends all the way out to the Centaurus-Hydra complex. The latter is located in what appears to be the intersection of the supergalactic plane with the TPI wall. Curiously, this intersection is located in the general direction of the Great Attractor (GA), leading to the speculation that the GA may simply represent a local density enhancement resulting from the intersection of two sheets.

\section{MODERATELY DEEP SURVEYS}

Although the nearby samples have provided important information and have been extensively used for comparison with $\mathrm{N}$-body simulations, they are too shallow, as there is mounting evidence for the existence of voids as large as $6,000 \mathrm{~km} \mathrm{~s}^{-1}$, comparable to the effective depth of the magnitude 14.5 surveys. This pointed out the need to extend the nearby surveys in depth. Towards this end the CfA group 
has been carrying out a survey of galaxies in 6 slices in the northern hemisphere down to $m_{\mathrm{B}}(0)<15.5$. So far they have completed 5 slices in the interval $12^{\circ}<\delta<42^{\circ}$, and 5 slices in the interval $9^{\circ}<\delta$ $<33^{\circ}$ in the southern galactic cap. The preliminary results have been outstanding, convincingly demonstrating the existence of thin sheet (wall)-like structures, as exemplified by the Great Wall, and the frequent occurrence of large, $6,000 \mathrm{~km} \mathrm{~s}^{-1}$ in diameter, voids (e.g. Geller and Huchra 1989). Similar work is being pursued in the southern hemisphere where a large collaborative effort has been organized to guarantee the rapid progress of the magnitude 15.5 survey (da Costa et al. 1989, da Costa 1991). Despite the difficulties in creating a deep galaxy sample, the SSRS2 is progressing well, and by the end of the current year we expect to have complete redshift information for galaxies over $30^{\circ}$ of the southern hemisphere in the region $-40^{\circ}<\delta<-10^{\circ}, b<-40^{\circ}$. The southern data will provide, in conjunction with that available in the north, a panoramic view of the galaxy distribution to greater depths, and independent samples and improved statistics to test the reliability of the statistical indicators used to describe galaxy clustering. The combined sample will also probe scales of the order of $300 h^{-1} \mathrm{Mpc}$ and be a better match to the new generation of $N$-body simulations becoming available.

\section{DEEP SURVEYS}

One example of the new generation deep surveys is the Century Survey being conducted by Geller et al. 1991 in the region $29^{\circ}<\delta<30^{\circ}$ and $8^{\mathrm{h}} 30^{\mathrm{m}}<\alpha<16^{\mathrm{h}} 30^{\mathrm{m}}$, for galaxies brighter than $m_{\mathrm{R}}=16.4$. The sample, which should include about 2,000 galaxies, is being generated from PDS scans of the Palomar plates and calibrated with CCD frames. The sample is sufficiently dense that most observations can be more efficiently carried out utilizing the Decaspec, a 10-fiber system mounted on the spectrograph of the MDM 2.4-m telescope. This survey is intended to be complete, measuring radial velocities for all galaxies brighter that the specified magnitude limit, with an effective depth of 30,000 $\mathrm{km} \mathrm{s}^{-1}$. Currently redshifts are available for 60 to $70 \%$ of the sample.

A second example of a wide-angle deep survey is the ongoing work of Kirshner et al. 1991, a sparse survey to about the same $30,000 \mathrm{~km} \mathrm{~s}^{-1}$ depth, but covering a large solid angle. Following a strategy similar to their earlier work, small fields of $1.5^{\circ} \times 3^{\circ}$ arranged in a grid were selected for photometry and redshift measurements in two separate regions: one in the southem galactic cap $\left(-48^{\circ}<\delta<-30^{\circ}, 21^{\mathrm{h}}<\right.$ $\left.\alpha<4^{\mathrm{h}} 30^{\mathrm{m}}\right)$ and the other in the northern galactic cap $\left(-18^{\circ}<\delta<0^{\circ}, 10^{\mathrm{h}}<\alpha<15^{\mathrm{h}}\right)$. Within these fields observations are made of 50 galaxies at a time, corresponding to the number of fibers currently available. So far a total of over 5,000 galaxies in the magnitude range $16<m_{\mathrm{R}}<17.3$ have been observed, in the two regions. In one case an entire slice of contiguous fields has been completed and the resulting redshift map provides a remarkable view of the galaxy distribution showing a void-filled universe with a characteristic scale of about $6,000 \mathrm{~km} \mathrm{~s}^{-1}$, with no evidence for structures on larger scales. This suggests that we may have finally reached a fair sample.

\section{VERY DEEP SURVEYS}

Complementing the surveys described above, exciting new data are also becoming available from pencil-beam surveys currently being conducted down to a limiting magnitude of about $m_{\mathrm{J}}=20$ to 21.5 (e.g. Broadhurst et al. 1990), corresponding to a depth of over 1,500 $h^{-1} \mathrm{Mpc}$. The preliminary results of the survey along the galactic poles are remarkable, as they suggest that the galaxy distribution exhibits a quasi-periodic pattern with a characteristic scale of about $120 \mathrm{~h}^{-1} \mathrm{Mpc}$. The sample along the galactic pole direction has been expanded with with velocity measurements of 700 additional galaxies (300 in the NGP direction and 400 in the SGP direction) bringing the total of available redshifts within a few degrees of the NGP-SGP probe to 1,500 galaxies. Remarkably, the new data seem to support the original claim of a quasi-periodic pattern. 
Two other directions are also being examined (Koo 1991). One of these is $45^{\circ}$ from the pole in the SA68/anti-SA68 direction, where 670 galaxies have measured redshifts. The data appear to be consistent with the pole data, showing a spike in the power-spectrum near $130 \mathrm{~h}^{-1} \mathrm{Mpc}$. However, the quasi-periodicity is less dramatic in this direction. Observations are also being carried out to probe a third direction, $90^{\circ}$ away from the SA68 direction, but only about 200 redshifts are currently available.

\section{CONCLUSIONS}

As briefly discussed, important new and complementary results can be expected for the next few years from the ongoing redshift surveys described here. Moreover, the development of large-format CCD detectors and the more frequent use of multi-fiber spectrographs should greatly increase the rate of data acquisition for both nearby and deep surveys. There are also exciting perspectives for future surveys based on new telescopes such as: the Princeton-Chicago million-redshift machine and the southern Arecibo-like telescope with its revolutionary design allowing for an $80^{\circ}$ sky coverage, broad band-width operation and multi-feed capabilities. If all goes well the 90 's may be just as exciting as the 80 's.

\section{REFERENCES}

Broadhurst, T. J., Ellis, R. S., Koo, D. C., and Szalay, A. S., 1990. Mon. Not. R. Astron. Soc. 343, 726.

da Costa, L. N., 1991. In The Proceedings of the Second DAEC Meeting, The Distribution of Matter in the Universe, eds. D. Gerbal and G. Mamon, in press.

da Costa, L. N., Pellegrini, P. S., Willmer, C. N. A., and Latham, D. W., 1989. Astrophys. J. 344, 20.

da Costa, L. N., Pellegrini, P. S., Davis, M., Meiksin, A., Sargent, W. L. W., and Tonry, J., 1991. Astrophys. J. Suppl. 76, 935.

Dressler, A., 1991. Astrophys. J. Suppl. 75, 241.

Fairall, A. P., Latham, D. W., Calderon, J. H., Willmer, C. N. A., da Costa, L. N., Pellegrini, P. S., Nunes, M. A., Focardi, P., and Vettolani, G., 1991. Astron. J. in press.

Geller, M. J., and Huchra, J. P., 1989. Sci. 246, 897.

Geller, M. J., Kurtz, M., Fabricant, D., Schild, R., Huchra, J. P., Thorstensen, J. R., and Wegner, G., 1991. Private communication.

Haynes, M. P., and Giovanelli, R. 1986. Astrophys. J. (Lett.) 306, L55.

Huchra, J. P., Davis, M., Latham, D. W., and Tonry, J. L., 1983. Astrophys. J. Suppl. 52, 89.

Huchra, J. P., Latham, D. W., da Costa, L. N., Pellegrini, P. S., and Willmer, C. N. A., 1991. In preparation.

Kirshner, R. P., Oemler, A. E., Schechter, P. L., Shectman, S. A., 1991. Private communication.

Koo, D., 1991. Private communication.

Tully, R. B., and Fisher, J. R., 1987. Nearby Galaxy Atlas, Cambridge: Cambridge University Press.

Willmer, C. N. A., Pellegrini, P. S., da Costa, L. N., Fairall, A. P., Focardi, P., Latham, D. W., and Calderon, J. H., 1991. In preparation. 